\title{
Immunotherapy and chimeric antigen receptor T-cell therapy in hepatocellular carcinoma
}

\author{
Pedro Luiz Serrano Uson Junior ${ }^{1 \#}$, Alex J. Liu ${ }^{2 \#}$, Mohamad Bassam Sonbol ${ }^{1}$, Mitesh J. Borad ${ }^{1}$, \\ Tanios S. Bekaii-Saab ${ }^{1}$ \\ ${ }^{1}$ Mayo Clinic Cancer Center, Phoenix, AZ, USA; ${ }^{2}$ Mayo Clinic Internal Medicine Residency, Phoenix, AZ, USA \\ Contributions: (I) Conception and design: PLS Uson Junior, AJ Liu, MB Sonbol; (II) Administrative support: MJ Borad, TS Bekaii-Saab; (III) \\ Provision of study materials or patients: None; (IV) Collection and assembly of data: None; (V) Data analysis and interpretation: None; (VI) \\ Manuscript writing: All authors; (VII) Final approval of manuscript: All authors. \\ \#These authors contributed equally to this work. \\ Correspondence to: Tanios S. Bekaii-Saab, MD. Professor of Medicine, Division of Hematology and Medical Oncology, Mayo Clinic, 5881 East Mayo \\ Blvd, Phoenix, Arizona 85054, USA. Email: Bekaii-Saab.Tanios@mayo.edu.
}

\begin{abstract}
Advanced hepatocellular carcinoma (HCC) is a deadly disease. With increasing incidence of new cases over the last years multiple efforts have been made to ameliorate survival and quality of life. Recent advances in understanding the tumor microenvironment and cancer immune evasion led to development of potent immune therapies targeting programmed death-ligand-1 (PD-L1), programmed cell death protein 1 (PD-1) and anti-cytotoxic T-lymphocyte-associated protein-4 (CTLA-4). Early clinical studies highlighted the activity and synergism of checkpoint inhibitors with antiangiogenic drugs, including antivascular endothelial growth factor (VEGF) antibodies and multi-tyrosine kinase inhibitors. Most recently, the combination of bevacizumab and atezolizumab improved survival compared to sorafenib in the treatment of advanced HCC on first-line therapy in a randomized phase III trial and now is considered the standard of care. Multiple options are available for the treatment of metastatic HCC including atezolizumab, bevacizumab, nivolumab, pembrolizumab, ipilimumab, sorafenib, lenvatinib, regorafenib, cabozantinib and ramucirumab. Furthermore, other checkpoint inhibitors are being evaluated including durvalumab, tremelimumab, CS1003, sintilimab and camrelizumab. In this review article, we focus on the landscape of different immunotherapy strategies in the management of HCC and the combination of checkpoint inhibitors antibodies with antiangiogenics. In addition, we will address the limitation of cell therapies in advanced HCC and current strategies to improve efficacy.
\end{abstract}

Keywords: Hepatocellular carcinoma (HCC); checkpoint inhibitors; programmed death-ligand-1 (PD-L1)

Submitted Nov 03, 2020. Accepted for publication Nov 20, 2020.

doi: $10.21037 / \mathrm{cco}-20-231$

View this article at: http://dx.doi.org/10.21037/cco-20-231

\section{Introduction}

Globally, liver cancer is estimated to be the sixth most diagnosed malignancy with over 840,000 new cases in 2018 $(1,2)$. Additionally, it is a relevant cause of mortality with 782,000 deaths $(1,2)$. Hepatocellular carcinoma (HCC) comprises $75-85 \%$ of primary liver cancers (2). The most common predisposing factors in HCC development include infections by hepatitis $\mathrm{B}$ virus (HBV) and $\mathrm{C}$ virus (HCV), alcohol use, aflatoxins, diabetes, and metabolic syndrome
$(3,4)$. HCC disproportionately affects Sub-Saharan Africa and Asia due to endemic chronic HBV infection (2). It is estimated that $~ 40 \%$ of HCC cases worldwide can be attributed to chronic HBV (4). However, there has been increasing incidence of HCC in traditionally low-risk HCC areas such as Western countries in the last twenty years, thought to be related to increased prevalence of obesity and non-alcoholic fatty liver disease (NAFLD) (5).

The overall five-year survival rate for HCC varies 
drastically depending on the stage at which the malignancy was originally diagnosed. If the disease is caught at its earlier stages (30-40\% of cases) and able to undergo curative measures with ablation, resection, or transplantation, median overall survival (OS) can be greater than 60 months (6). However, most cases (50-60\%) are diagnosed at advanced stages and managed with palliative intent, with a median OS of 26 months with chemoembolization in intermediate stage HCC, and only around 12 months in advanced HCC (6).

\section{Liver and tumor microenvironment}

The liver is constantly exposed to diverse dietary and microbial antigens from the gastrointestinal system through portal venous flow $(7,8)$. As a result, it contains large populations of resident macrophages (Kupffer cells), lymphocytes and natural killer (NK) cells, which respond to antigenic presentation through cytokine production and killing of targeted cells (9-11). However, the liver also establishes immune tolerance in instances of nonpathogenic stimuli to avoid chronic inflammation. Kupffer cells assist in immune regulation by secreting IL-10 to inactivate NK cells, induce T-regulatory cells and inhibit T cells through expression of programmed cell death protein 1 (PD-1) and programmed death-ligand-1 (PD-L1) $(8,10)$. Complex mechanisms of pro-inflammatory response and immune tolerance occur in the setting of fibrosis and chronic inflammation. The setting of chronic antigen stimulation leads to $\mathrm{T}$ cell exhaustion with upregulation of PD-1 and PD-L1 receptors $(8,11)$. Studies have found that HCC tissue have increased PD-1 expression in CD8+ and CD4+ $T$ cells associated with diminished cytotoxic function when compared with surrounding healthy tissue (12-14). PD-L1 expression has been strongly correlated with tumor size, poorer survival, and increased post-operative recurrence in HCC (15). The interplay of immunotolerance with fibrosis, chronic inflammation, and HCC development has spurred interest in novel therapies with checkpoint inhibitors (ICI).

\section{Immune checkpoint inhibitors clinical trials}

Checkmate-040, an open-label phase I/II dose expansion and escalation trial, evaluated nivolumab, an antibody antiPD-1, to assess safety and efficacy. Patients included had Child-Pugh scores of 7 or less, and an Eastern Cooperative Oncology Group (ECOG) performance status of 0 or 1 . Patients were treated with nivolumab $0.1-10 \mathrm{mg} / \mathrm{kg}$ every two weeks (q2w) in the dose-escalation phase and $3 \mathrm{mg} / \mathrm{kg}$ $\mathrm{q} 2 \mathrm{w}$ in the dose-expansion phase. There were four cohorts of patients: sorafenib untreated or intolerant without viral hepatitis, sorafenib progressors without viral hepatitis, and cohorts associated with HCV or HBV infections (16). During the dose-escalation phase, nivolumab showed an acceptable safety profile. An objective response was seen in 42 of $214(20 \%)$ patients in the dose-expansion cohort, with a median duration of response of 9.9 months. Stable disease was observed in 96 (45\%) patients (16). Similarly, in KEYNOTE-224, a non-randomized open-label phase 2 study, pembrolizumab (PD-1 inhibitor) was utilized in Child-Pugh A patients with advanced HCC previously exposed to sorafenib. One hundred and four eligible patients were enrolled and treated with pembrolizumab $200 \mathrm{mg}$ every three weeks $(\mathrm{q} 3 \mathrm{w})$ until two years or progression of disease. Objective response was observed in 18 (17\%; 95\% CI, 11-26\%) patients with median duration not reached (3.1-14.6+ months). Disease stability occurred in 46 (44\%) patients, and disease progression in 34 (33\%) patients. Treatment-related adverse events (TRAE) occurred in 76 (73\%) of 104 patients, with grades $1-2$ in 49 (47\%) patients, grade 3 in 25 (24\%), grade 4 in one patient and grade 5 in one patient. Fatigue, elevated aspartate aminotransferase, pruritus, rash, and diarrhea were among the most common TRAE. Expression of PD-L1 was assessed in 52 patients, finding that the objective responses were $32 \%$ (7 of 22 patients) with combined positive scores of at least 1 and $20 \%$ (6 of 30 patients) with combined positive scores less than 1 (17). These studies led to the US Food and Drug Administration (FDA) accelerated approval of nivolumab and pembrolizumab monotherapies in patients with advanced HCC.

Another anti-PD-1, camrelizumab, was evaluated in an open-label, parallel, randomized trial in patients with advanced HCC (18). Two hundred and seventeen patients with advanced HCC previously exposed to systemic treatment were evaluated in two groups, camrelizumab $3 \mathrm{mg} / \mathrm{kg}$ every two or three weeks. In a median follow-up of 12.5 months, objective response was observed in 32 patients (14.7\%; 95\% CI, 10.3-20.2\%). The OS rate in 6-month was $74.4 \%$ (95\% CI, 68.0-79.7\%). Median PFS for all patients treated was 2.1 months (95\% CI, 2.0-3.2), and median OS was 13.8 months (95\% CI, 11.5-16.6). Adverse events related to treatment were found in $>20.0 \%$, with reactive cutaneous capillary endothelial proliferation (RCCEP) as the most common immune-related adverse event (67\%, all grade $\leq 2)$. Of note, those who had experienced RCCEP had 
significantly higher objective response (19.3\% vs. 5.6\%) (18).

Despite the early enthusiasm with PD-1 inhibitors, later phase 3 data questioned the efficacy of such agents as monotherapies in HCC. Results from CheckMate-459 that compared nivolumab to sorafenib in patients with advanced HCC in the first-line setting were presented (19). Seven hundred and forty-three patients were randomized to nivolumab $(\mathrm{n}=371)$ or sorafenib $(\mathrm{n}=372)$. ORR was higher in nivolumab (15\%) compared to sorafenib (7\%). However, despite median OS of nivolumab was numerically higher than sorafenib, 16.4 versus 14.7 months, the primary endpoint did not reach statistical significance (HR 0.85; 95\% CI, 0.72-1.02; P=0.0752) (19). Sorafenib accounted for more grade $3 / 4$ TRAE (49\% vs. 22\%) and treatment discontinuation ( $8 \%$ vs. 4\%) (19). Similarly, randomized phase III trial evaluating pembrolizumab in the secondline setting did not meet its primary end point (20). In KEYNOTE-240, total of 413 patients were assigned in a 2:1 ratio to the pembrolizumab group $(n=278)$ or placebo group $(\mathrm{n}=135)$. ORR in the pembrolizumab group was $18.3 \%$ compared with $4.4 \%$ in the placebo group. The median OS of pembrolizumab group was 13.9 months compared with 10.6 months in the placebo group (HR $0.781 ; 95 \%$ CI, 0.611-0.998; $\mathrm{P}=0.0238)$. Additionally, median progression-free survival (PFS) was 3.0 months for the pembrolizumab group compared with 2.8 months for placebo (HR 0.718; 95\% CI, 0.570-0.904; $\mathrm{P}=0.0022$ ). Despite the improvements in OS and PFS compared with placebo, prespecified parameters of $\mathrm{P}=0.0174$ for $\mathrm{OS}$ and $\mathrm{P}=0.002$ for PFS were not reached (20). Both these trials, CheckMate-459 in first-line setting and KEYNOTE-240 in second-line setting, showed that probably PD-1 inhibitors as monotherapy have limited activity in the majority of unselected HCC patient population, emphasizing the importance of better biomarker selection in this disease.

Tremelimumab, a monoclonal antibody that binds T-lymphocyte-associated protein 4 (CTLA-4) was initially studied in a phase II trial, in 20 patients with HCC and chronic HCV infection. There was an observed disease control rate (DCR) of 76\%, partial response rate (PR) $17.6 \%$ with median time to progression (TTP) 6.4 months (95\% CI, 3.95-9.14) and median OS 8.2 months (95\% CI, 4.64-21.34). The drug had no safety concerns, with skin rash as the most common adverse event (65\%). Forty-five percent of patients had grade 3 transaminase elevations, although this was transient and did not require steroids (21). Tremelimumab was later studied in conjunction with radiofrequency ablation, chemoablation, or transarterial chemoembolization (TACE) in advanced HCC. A total of 32 patients were involved. Five in 19 patients with lesions outside of areas treated with ablation/TACE had partial response (PR). TTP was 7.4 months (95\% CI, 4.7-9.4 months), PFS rate in 6-month was $57.1 \%$ (95\% CI, 37.1-72.9\%) and in 12 -month was $33.1 \%$ (95\% CI, 16.2-51.2\%). Median OS was 12.3 months (95\% CI, 9.3-15.4 months). Given that all patients had also being treated with tremelimumab, with no control group, it was not clear if improvements were associated with the ablation procedure (22).

Association of anti-PD1 antibodies and anti-cytotoxic T-lymphocyte-associated protein 4 (CTLA-4) antibodies is based on the probable action of recruiting lymphocytes with the combined inhibition of B7-CTLA4 pathway. The efficacy of anti-PD-1 antibodies would be improved with the higher concentration of activated lymphocytes CD8+ in the tumor microenvironment $(23,24)$. In a phase I/II trial, the combination of durvalumab, an anti-PD-L1 antibody, with tremelimumab, $(\mathrm{D}+\mathrm{T})$ was evaluated (25). A total of 40 patients with advanced HCC were exposed to the regimen. Overall DCR was $57.5 \%$ and objective RP 15\% (25). Expansion cohorts further evaluated these drugs in different doses, in a four-arm study. A total of 332 patients who progressed, were intolerant, or refused sorafenib were randomized to single agent durvalumab, single agent tremelimumab or one of the two D+T combinations (tremelimumab $300 \mathrm{mg}+$ durvalumab 1,500 $\mathrm{mg}$ followed by durvalumab every 4 weeks or tremelimumab $75 \mathrm{mg}+$ durvalumab $1,500 \mathrm{mg}$ followed by durvalumab every 4 weeks). The RPs and median OS were: $7.2 \%$ and 17.1 months in tremelimumab group, $9.6 \%$ and 11.7 months in durvalumab group, $9.5 \%$ and 11.3 months in tremelimumab $75 \mathrm{mg}+$ durvalumab 1,500 $\mathrm{mg}$ combination group, and was the highest in the tremelimumab $300 \mathrm{mg}+$ durvalumab $1,500 \mathrm{mg}$ group with ORR of $22.7 \%$ and median OS of 18.7 months. However, this combination (T300+D) was associated with the highest toxicity profile with $35 \%$ of patients developing grade $3 / 4$ TRAEs and $10.8 \%$ discontinuing the treatment due to adverse events. A randomized phase III trial HIMALAYA (NCT03298451) is evaluating $\mathrm{D}+\mathrm{T}$ combination therapy against sorafenib in the first-line treatment of patients with advanced HCC, the study finished recruiting patients and results are eagerly awaited (26).

Similar to the trend above with increased efficacy when using higher doses of CTLA-4 inhibitors was found with ipilimumab. Ipilimumab, another anti-CTLA-4 antibody, was evaluated in association with nivolumab in 
the Checkmate 040 study. The combination was evaluated in three arms with different doses, arm A was nivolumab $1 \mathrm{mg} / \mathrm{kg}$ plus ipilimumab $3 \mathrm{mg} / \mathrm{kg}$ every three weeks (q3w) for 4 doses, followed by nivolumab $240 \mathrm{mg} \mathrm{q} 2 \mathrm{w}$, arm B nivolumab $3 \mathrm{mg} / \mathrm{kg}$ plus ipilimumab $1 \mathrm{mg} / \mathrm{kg} \mathrm{q} 3 \mathrm{w}$ (4 doses), followed by nivolumab $240 \mathrm{mg} \mathrm{q} 2 \mathrm{w}$, or arm C nivolumab $3 \mathrm{mg} / \mathrm{kg} \mathrm{q} 2 \mathrm{w}$ plus ipilimumab $1 \mathrm{mg} / \mathrm{kg}$ every six weeks $(\mathrm{q} 6 \mathrm{w})$. Of the 148 patients randomized, high proportion of patients presented with adverse prognostic features including vascular invasion and extrahepatic spread, $9 \%$ had at least 3 previous systemic treatments for advanced disease. The ORR was $32 \%(8 \% \mathrm{CR})$ in arm $\mathrm{A}$, and $31 \%(6 \% \mathrm{CR})$ in arm $\mathrm{B}$, and $31 \%(0 \% \mathrm{CR})$ in arm C. Median OS in arm A was 22.8 months (9.4-not reached), 12.5 months in arm $\mathrm{B}$, and in arm $\mathrm{C}$ was 12.7 months. Of note, this study was not powered to evaluate efficacy differences between each treatment arms. A total of $53 \%$ of patients had a grade 3-4 TRAE in arm A, most commonly with autoimmune hepatitis, rash, diarrhea, and colitis. TRAE led to drug discontinuation in $18 \%$ of patients in arm A, $6 \%$ and $2 \%$ of patients in arm B and C, respectively. The majority of TRAE resolved across all arms with corticosteroids being used in higher grades (27). The results led to the approval by the FDA of the combination in patients who have progressed on prior sorafenib. A summary of the early studies and results can be found on Table 1. The above data shows the increased efficacy with combining programmed cell death inhibitors with higher doses of CTLA-4 inhibitors antibodies. However, as this associated with significant toxicity, further level-1 evidence is needed to further establish the role of these combinations in advanced HCC.

\section{Combination of immune checkpoint inhibitors with antiangiogenics}

Pathways associated with vascular endothelial growth factor (VEGF) contribute to multiple immunosuppressive effects in tumor microenvironment $(28,29)$. The blockage of VEGF associated pathways is associated with normalization of tumor vasculature, decreased activation of myeloidderived suppressor cells (MDSCs), tumor-associated macrophages (TAMs) and regulatory $\mathrm{T}$ cells (Tregs), and improvements in maturation of dendritic cells resulting in an immunostimulatory microenvironment more accessible to immune checkpoint inhibitors $(28,29)$. Anti-PD-L1 atezolizumab with bevacizumab (anti-VEGF antibody) was first evaluated in a phase IB cohort of 68 advanced HCC patients. At a minimum follow-up of 18 weeks, ORR was $34 \%$, with responses observed in all clinically relevant subgroups. The combination had a manageable safety profile (30). Interestingly, in an exploratory post-roc analysis from GO30140 study showed that a subset of patients that experienced disease progression after atezolizumab for naïve advanced disease, had some benefit with addition of bevacizumab to atezolizumab in a crossover fashion (31). Most recently, IMbrave150 trial, a randomized phase III study, compared atezolizumab + bevacizumab with sorafenib in 366 treatment-naïve advanced HCC patients (32). In this study, patients with ECOG $0-1$ and Child-Pugh A were randomized in a 2:1 ratio to receive the combination or sorafenib. Overall, more than $70 \%$ of patients included were Child-Pugh A5, 62\% had an ECOG performance status 0 , and $48 \%$ of patients treated in the combination group had prior local liver directed therapies. It was found that OS HR was 0.58 (95\% CI, 0.42-0.79; $\mathrm{P}=0.0006)$ and PFS 6.8 months with atezolizumab + bevacizumab versus 4.3 months with sorafenib (HR 0.59; 95\% CI, 0.47-0.76; $\mathrm{P}<0.0001)$. The estimated median OS with sorafenib was 13.2 months compared to median that was not reached in the combination arm. Additionally, adverse event rates were comparable, with grade 3-4 events occurring in $57 \%$ of patients with the combined regimen compared with $55 \%$ of patients who received sorafenib. The results of this study led to the FDA approval of the combination as standard of care (32). Although the combination (atezolizumab/ bevacizumab) was not directly compared with other firstline treatments, a recent network meta-analysis showed the superiority of this regimen compared with lenvatinib and nivolumab as well (33).

Similarly, lenvatinib, a multi-kinase inhibitor that targets VEGFR1-3, was also evaluated with pembrolizumab in a phase Ib trial in advanced HCC (34). In this study the combination was evaluated in 100 patients in the first-line setting of systemic treatment. Median OS was 22 months (95\% CI, 20.4-not estimable) and the median PFS was 8.6 months (95\% CI, 7.1-9.7). The ORR by RECIST v1.1 per independent image review was $36 \%$. In this cohort, $95 \%$ of patients (grade $\geq 3,67 \%$; grade $\geq 4,4 \%$ ) presented TRAE. Three patients died from TRAE (34). This early promising success led to an FDA breakthrough therapy designation to the combination in July 2019. However, the FDA recently held off on the originally planned approval of pembrolizumab/lenvatinib as the combination no longer meets the criteria for accelerated approval of showing meaningful improvement over available 
Table 1 Results from early clinical studies including immune checkpoint inhibitors in advanced hepatocellular carcinoma

\begin{tabular}{|c|c|c|c|c|c|c|c|c|}
\hline Study & Design & Drug & $\mathrm{N}^{\circ}$ & Characteristics & ORR & DCR & mPFS (mo) & $\mathrm{mOS}(\mathrm{mo})$ \\
\hline $\begin{array}{l}\text { El-Khoueiry } \\
\text { et al. } 2017 \text { (16) }\end{array}$ & Phase I/II & Nivolumab & 262 & $\begin{array}{l}\text { Child-Pugh A or B; } \\
\text { Naïve or exposed }\end{array}$ & $20 \%$ & $64 \%$ & - & - \\
\hline $\begin{array}{l}\text { Kelley et al. } \\
2017 \text { (25) }\end{array}$ & Phase I & $\begin{array}{l}\text { Durvalumab + } \\
\text { Tremelimumab }\end{array}$ & 40 & $\begin{array}{l}\text { Child-Pugh A; } \\
\text { Naïve or exposed }\end{array}$ & $15 \%$ & $57.5 \%$ & - & - \\
\hline $\begin{array}{l}\text { Zhu et al. } \\
2018 \text { (17) }\end{array}$ & Phase II & Pembrolizumab & 104 & $\begin{array}{c}\text { Child-Pugh A; } \\
\text { Previous sorafenib }\end{array}$ & $17 \%$ & $62 \%$ & 4.9 & 12.9 \\
\hline \multirow{2}{*}{$\begin{array}{l}\text { Yau et al. } \\
2020 \text { (27) }\end{array}$} & Phase II & NIVO1+IPI3 Q3W & 50 & \multirow{2}{*}{$\begin{array}{l}\text { Child-Pugh A; } \\
\text { Prior sorafenib }\end{array}$} & $32 \%$ & $54 \%$ & - & 22.8 \\
\hline & & NIVO3+IPI1 Q3W & 49 & & $27 \%$ & $43 \%$ & & 12.5 \\
\hline \multirow{4}{*}{$\begin{array}{l}\text { Kelley et al. } \\
2020 \text { (26) }\end{array}$} & \multirow[t]{4}{*}{ Phase II } & Durvalumab & 104 & \multirow{4}{*}{$\begin{array}{l}\text { Child-Pugh A; } \\
\text { Prior sorafenib }\end{array}$} & $9.6 \%$ & \multirow[t]{4}{*}{-} & \multirow[t]{4}{*}{-} & 11.7 \\
\hline & & Tremelimumab & 69 & & $7.2 \%$ & & & 17.1 \\
\hline & & Tremelimumab $300 \mathrm{mg}+\mathrm{D}$ & 75 & & $22.7 \%$ & & & 18.7 \\
\hline & & Tremelimumab $75 \mathrm{mg}+\mathrm{D}$ & 84 & & $9.5 \%$ & & & 11.3 \\
\hline
\end{tabular}

$\mathrm{N}^{\circ}$, number of patients; ORR, objective response rate; DCR, disease control rate; mo, months; mPFS, median progression-free survival; mOS, median overall survival; D, durvalumab; NIVO1, nivolumab 1 mg/kg; NIVO3, nivolumab 3 mg/kg; IPI1, ipilimumab 1 mg/kg; IPI3, ipilimumab 3 mg/kg; Q2W, every 2 weeks; Q3W, every 3 weeks; Q6W, every 6 weeks; Anti-PD1: Nivolumab, Pembrolizumab, Camrelizumab; Anti-PD-L1: Durvalumab; Anti-CTLA-4: Ipilimumab, Tremelimumab.

therapies (atezolizumab/bevacizumab). Further data is awaited with the phase 3 LEAP 002 trial (NCT03713593) comparing sorafenib with pembrolizumab/lenvatinib combination in advanced treatment naïve HCC. The study is currently ongoing but not enrolling any additional patients. Pembrolizumab plus lenvatinib and transarterial chemoembolization (TACE) will also be evaluated in a randomized phase 3 trial for intermediate HCC ineligible for curative treatment (35). In addition, lenvatinib is being evaluated associated with nivolumab in the IMMUNIB trial (36). Data from safety in this investigator-initiated phase II trial were presented in ESMO 2020, no limiting toxicities were observed in both cohorts with nivolumab $240 \mathrm{mg}$ plus lenvatinib 12 or $8 \mathrm{mg}$. The study is still recruiting patients (36). Another anti-PD-1, CS1003, is being evaluated combined with lenvatinib. Sixteen Chinese patients with advanced HCC Child-Pugh A received the combination and were evaluated for efficacy in the phase Ib study. The objective RP was $37.5 \%$. Like other studies TRAE was observed in $84.2 \%$ of the patients (37).

Another multi-kinase inhibitor cabozantinib, blocks
VEGFR 1-3, AXL, c-MET and RET, was evaluated combined with nivolumab and ipilimumab in Checkmate 040 study. Seventy-one patients were randomized in two arms: 36 patients were treated with nivolumab $240 \mathrm{mg}$ q2 $\mathrm{w}$ plus cabozantinib $40 \mathrm{mg}$ daily and 35 patients were treated with nivolumab $3 \mathrm{mg} / \mathrm{kg}$ q2 $\mathrm{w}$ plus ipilimumab $1 \mathrm{mg} / \mathrm{kg}$ q6w and cabozantinib $40 \mathrm{mg}$ daily. ORR was $17 \%$ in the nivolumab plus cabozantinib arm with a DCR of $81 \%$. The RP was $26 \%$ in the nivolumab, ipilimumab plus cabozantinib arm with a DCR of $83 \%$. Median OS is still not reached. It is important to note that in these cohorts, prior sorafenib exposure was allowed (38).

Regorafenib, another multiple tyrosine-kinase inhibitor with antiangiogenic features is also evaluated combined with anti-PD-1 for first-line systemic treatment of patients with metastatic HCC. The combination pembrolizumab and regorafenib was evaluated for safety and tolerability in a dose-finding study (39). In 18 patients treated with regorafenib $120 \mathrm{mg}$, four presented dose-limiting toxicities including transaminases and bilirubin elevations, and rash. Of 32 patients evaluated for response, $28 \%$ had a partial 
response, with a DCR of $91 \%(39)$.

A phase $2 / 3$ is being conducted to evaluate combination of sintilimab, an anti-PD-1 antibody, and IBI305, a bevacizumab biosimilar, versus sorafenib for the treatment of patients with advanced HCC. Data from phase II part of the trial were presented, with 24 patients enrolled. After a median follow-up of 9.0 months, the median PFS in patients treated with the combination was 8.4 months (95\% CI, 5.6-not reached), the ORR was $25 \%$ and the DCR $83.3 \%$, accessed by the investigator. The randomized phase 3 trial evaluating the combination is ongoing, with a planned recruitment accrual of 546 patients (40).

Camrelizumab (SHR-1210) and apatinib (VEGFR2 inhibitor) were studied in an open-label phase Ia and Ib to assess tolerability and clinical activity in patients with HCC and gastroesophageal cancers (41). For dose escalation portion, 5 patients per cohort received apatinib in oral doses of 125,250 , or $500 \mathrm{mg}$ with camrelizumab $200 \mathrm{mg}$ (administered intravenously every 2 weeks). Fifteen patients (HCC $\mathrm{n}=8)$ were in the dose escalation phase and 28 patients ( $\mathrm{HCC} \mathrm{n}=10$ ) were in the subsequent dose expansion phase (apatinib $250 \mathrm{mg}$ was the selected dose). Out of 16 evaluable HCC patients, 8 achieved PR. ORR was $50.0 \%$ (95\% CI, 24.7-75.4\%) and DCR was 93.8\% (95\% CI, 69.8-99.8\%). Median time to response was 3.4 months (1.4-9.7 months) and median PFS was 5.8 months (95\% CI: 2.5-not reached). Treatment was well-tolerated at the apatinib $250 \mathrm{mg}$ dose, with grade $\geq 3$ TRAE most commonly being hypertension (15.2\%) and elevated aspartate aminotransferase (15.2\%). Of note, PD-L1 levels were evaluated on circulating tumor cells (CTCs) in 35 patients, and it was found that patients with $\geq 20 \%$ PDL $1{ }^{\text {high }}$ CTCs had a significantly longer PFS compared with $<20 \%$ PD-L1 ${ }^{\text {high }}$ CTCs (6.1 vs. 2.9 months, HR 0.28; 95\% CI, 0.12-0.67; P=0.0002). This study suggested that apatinib and camrelizumab were well tolerated and led to clinical efficacy, particularly in patients with $\geq 20 \%$ PD-L1 ${ }^{\text {high }}$ CTCs at baseline (41). Further data from the combination on first-line and second-line of treatment for advanced disease was presented (42). This study involved patients from 25 study sites in China. Patients were treatment-naïve or sorafenib or donafenib exposed. Seventy treatment naïve patients and 120 patients previously treated (second-line setting) were enrolled to receive camrelizumab and apatinib, $88 \%$ were patients with $\mathrm{HBV}$ infection. The objective response assessed by independent central review per RECIST v1.1 was 34\% and 23\%; with a 12 -month OS rate of $75 \%$ and $68 \%$, respectively. Overall, $77 \%$ of patients had grade $\geq 3$ TRAEs, with hypertension and increased $\gamma$-GT as common adverse events (42). A summary of the early studies and results with immune checkpoint inhibitors and antiangiogenics can be found on Table 2. Data from randomized phase 3 trials can be found in Table 3 .

\section{Chimeric antigen receptor T-cell (CAR-T)/T-cell receptors (TCR) therapy}

CAR-T therapy is another avenue through which the immune system can be harnessed to target a tumor. By combining an extracellular ligand recognition domain with an intracellular signaling platform, these synthetically developed receptors in $\mathrm{T}$ cells can be activated by specific tumor antigens $(43,44)$. CAR-T therapy has found success in B cell malignancies through targeting of CD19; however, testing in solid tumors has not yielded viable options as of yet. The lack of efficacy in solid tumors can be attributed to utilizing suboptimal tumor antigens as targets, as well as the inhibitory nature of the tumor microenvironment. Within the tumor microenvironment, hypoxia, nutrient deprivation, and expression of inhibitory checkpoint ligands stymie the proliferation and effector function of $\mathrm{T}$ cells $(45,46)$. Tumor production of indoleamine 2,3-dioxygenase (IDO) catalyzes tryptophan breakdown and resultant formation of metabolites that have been found to inhibit CAR-T cells (47).

Additionally, while efficacy of CAR-T therapy in solid tumors has been limited, significant adverse effects have been associated with CAR-T which include cytokine release syndrome and neurotoxicity $(48,49)$. Thus, selecting highly specific HCC antigens would be key to delivering a more efficacious and safe therapy.

Glypican-3 (GPC3), a heparin sulfate proteoglycan highly expressed in most HCC has been explored as a target for CAR-T therapy. There have been several studies that showed activity against HCC in vivo and in patientderived xenografts with anti-GPC3 CAR-T therapy (50-52). Of note, it was found that the GPC3 CAR-T cells were able to eradicate PD-L1 negative tumors in xenografts, whereas the PD-L1 tumors were more aggressive (51), and models demonstrate that generated PD-1-deficient GPC3 CAR-T cells exhibits 1.25-1.30 times the cytotoxic activity of wild-type GPC3 CAR-T cells (51-53). These findings suggest the potential of combination of immune checkpoint inhibitors to enhance CAR-T therapy's cytotoxic effects in $\operatorname{HCC}(54,55)$.

Alpha-fetoprotein (AFP) is used as biomarker for hepatocellular carcinoma, with $60 \%$ to $80 \%$ of the tumors 
Table 2 Results from early clinical studies including immune checkpoint inhibitors with antiangiogenics

\begin{tabular}{|c|c|c|c|c|c|c|c|c|}
\hline Study & Design & Drug & $\mathrm{N}^{\circ}$ & Characteristics & ORR & DCR & mPFS (mo) & $\mathrm{mOS}(\mathrm{mo})$ \\
\hline Zhu et al. 2020 (34) & Phase lb & Pembrolizumab + Lenvatinib & 100 & $\begin{array}{l}\text { Child-Pugh A; } \\
\text { Treatment naïve }\end{array}$ & $36 \%$ & $88 \%$ & 8.6 & 22.0 \\
\hline $\begin{array}{l}\text { Galle et al. } 2020 \\
\text { (39) }\end{array}$ & Phase lb & $\begin{array}{c}\text { Pembrolizumab + } \\
\text { Regorafenib }\end{array}$ & 32 & $\begin{array}{l}\text { Child-Pugh A; } \\
\text { Treatment naïve }\end{array}$ & $28 \%$ & $91 \%$ & - & - \\
\hline Jia et al. 2020 (40) & Phase II & Sintilimab + IBI305 & 24 & $\begin{array}{l}\text { Child-Pugh A; } \\
\text { Treatment naïve }\end{array}$ & $25 \%$ & $83.3 \%$ & 8.4 & NE \\
\hline Xu et al. 2020 (42) & Phase II & Camrelizumab + Apatinib & 70 & Treatment naïve & $34 \%$ & $77 \%$ & 5.7 & - \\
\hline Yau et al. 2020 (38) & & $\begin{array}{c}\text { Nivolumab + IPI + } \\
\text { Cabozantinib }\end{array}$ & 35 & Naïve or exposed & $26 \%$ & $83 \%$ & 6.8 & NE \\
\hline
\end{tabular}

$\mathrm{N}^{\circ}$, number of patients; ORR, objective response rate; DCR, disease control rate; mo, months; mPFS, median progression-free survival; mOS, median overall survival; IPI, ipilimumab, NE, not estimable; Anti-PD-1: Nivolumab, Pembrolizumab, CS1003, Sintilimab, Camrelizumab; Anti-PD-L1: Atezolizumab; Anti-CTLA-4: Ipilimumab; Tyrosine kinase inhibitors: Lenvatinib, Cabozantinib, Regorafenib, Apatinib; VEGF inhibitors: Bevacizumab, IBI305.

Table 3 Results from randomized phase III studies of immune checkpoint inhibitors in advanced hepatocellular carcinoma

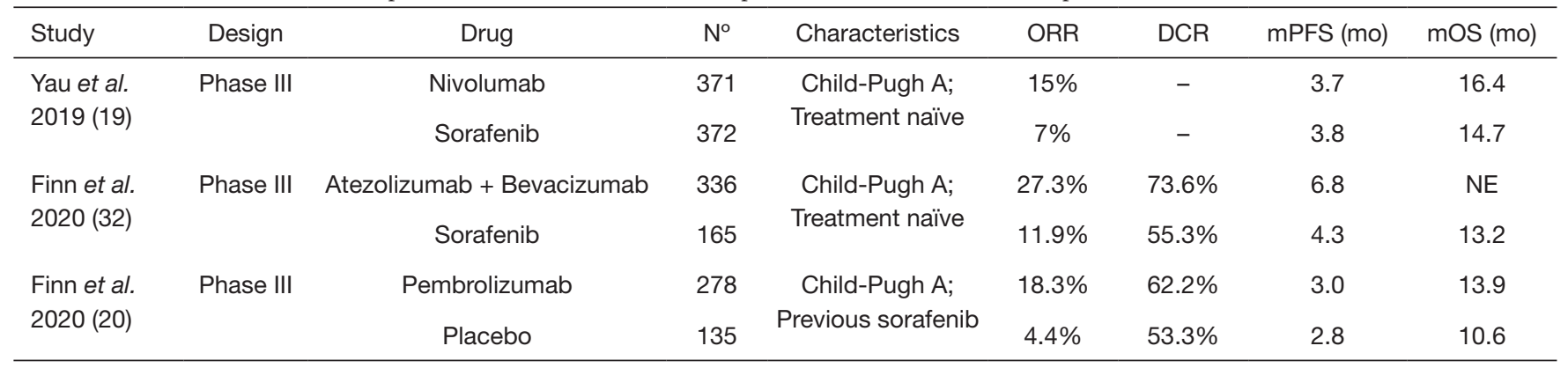

Anti-PD-1: Nivolumab, Pembrolizumab; Anti-PD-L1: Atezolizumab; Tyrosine kinase inhibitors: Sorafenib; VEGF inhibitors: Bevacizumab. $\mathrm{N}^{\circ}$, number of patients; ORR, objective response rate; $\mathrm{DCR}$, disease control rate; mo, months; mPFS, median progression-free survival; mOS, median overall survival.

expressing the protein (56). Tumor-specific T cells have low affinity, and an option to obtain high-affinity antigenspecific $\mathrm{T}$ cells is to genetically modify $\mathrm{T}$ cells with tumorspecific TCR (57-59). TCR developed to target AFP expressing HCC have been clinically evaluated with no major safety concerns (57-59). The interaction of TCRs with major histocompatibility complex (MHC) on the cell surface improved the affinity and is being evaluated in clinical trials, with most studies using human leukocyte antigen (HLA) A02:01 given prevalence and early results $(59,60)$. In Table 4 some CAR-T and TCRs studies in recruiting stage are exemplified.

\section{Conclusions}

In the setting of advanced HCC, immune-directed therapies are particularly novel and established a new paradigm in the treatment of the disease. New combinations with 
Table 4 Ongoing studies with CAR-T/TCR therapy in hepatocellular carcinoma

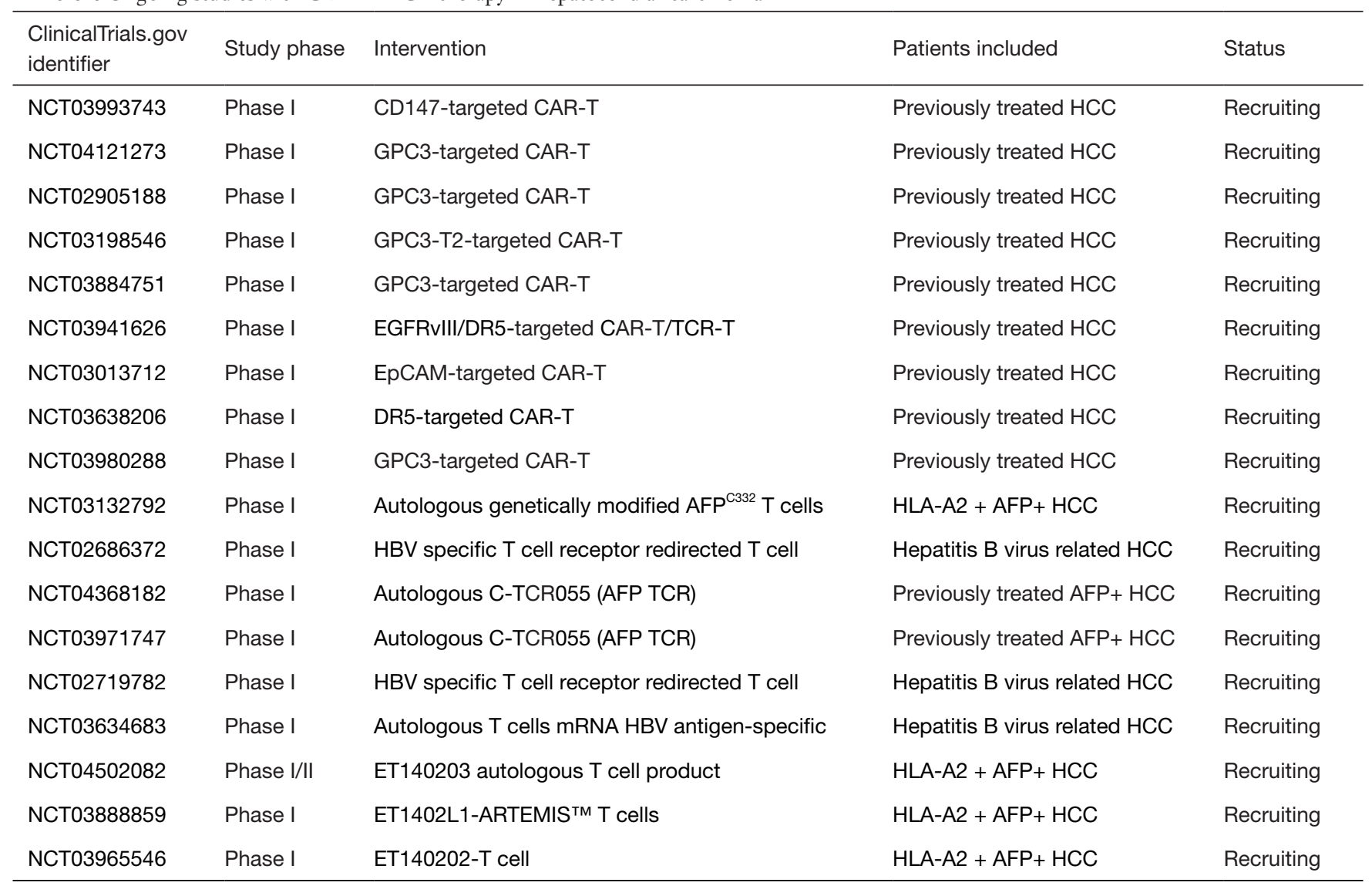

Ongoing clinical trials were identified by searching ClinicalTrials.gov using the terms "Hepatocellular carcinoma" and "CAR-T" and "TCR", on October $14^{\text {th }}$, 2020. CAR-T, chimeric antigen receptor T-cell; TCR, T-cell receptors.

antiangiogenics are emerging and standard of care with novel drugs could be achieved soon. Immune cell therapy associated with PD-1 pathway blockage could be a new revenue for the success of the strategy in solid tumors including advanced hepatocellular carcinoma.

\section{Acknowledgments}

Funding: None.

\section{Footnote}

Provenance and Peer Review: This article was commissioned by the Guest Editors (Mehmet Akce and Shishir K. Maithel) for the series "Hepatocellular Carcinoma" published in Chinese Clinical Oncology. The article was sent for external peer review organized by the Guest Editors and the editorial office.
Conflicts of Interest: The authors have completed the ICMJE uniform disclosure form (available at http://dx.doi. org/10.21037/cco-20-231). The series "Hepatocellular Carcinoma" was commissioned by the editorial office without any funding or sponsorship. Dr. MJB reports grants from Senhwa Pharmaceuticals, grants from Adaptimmune, grants from Agios Pharmaceuticals, grants from Halozyme Pharmaceuticals, grants from EMD Merck Serono, grants from Celgene Pharmaceuticals, grants from Toray, grants from Dicerna, grants from Taiho Pharmaceuticals, grants from Sun Biopharma, grants from Isis Pharmaceuticals, grants from Redhill Pharmaceuticals, grants from Boston Biomed, grants from Basilea, grants from Incyte Pharmaceuticals, grants from Mirna Pharmaceuticals, grants from Medimmune, grants from Bioline, grants from Sillajen, grants from ARIAD Pharmaceuticals, grants from PUMA Pharmaceuticals, grants from Novartis Pharmaceuticals, grants from QED Pharmaceuticals, grants 
from Pieris Pharmaceuticals, other from ADC Therapeutics, other from Exelixis Pharmaceuticals, other from Inspyr Therapeutics, other from G1 therapeutics, other from Immunovative, other from OncBioMune Pharmaceuticals, other from Western Oncolytics, other from Lynx Group, other from Astra Zenica, outside the submitted work. Dr. TSBS reports grants from Boston biomedical, grants and personal fees from Bayer, grants from Amgen, grants and personal fees from Merck, grants from Celgene, grants and other from Lilly, grants and personal fees from Ipsen, grants from Clovis, grants and personal fees from Seattle Genetics, grants and personal fees from Array biopharma, grants and personal fees from Genentech, grants from Abgenomics, grants and personal fees from Incyte, grants from BMS, personal fees from Boehringer Ingelheim, personal fees from TreosBio, personal fees from Sobi, other from Astra zenica, other from PanCan, other from 1Globe, other from Sun biopharma, other from Imugene, other from Immuneering, outside the submitted work. In addition, Dr. TSBS has a patent WO/2018/183488 issued, and a patent WO/2019/055687 issued. The authors have no other conflicts of interest to declare.

Ethical Statement: The authors are accountable for all aspects of the work in ensuring that questions related to the accuracy or integrity of any part of the work are appropriately investigated and resolved.

Open Access Statement: This is an Open Access article distributed in accordance with the Creative Commons Attribution-NonCommercial-NoDerivs 4.0 International License (CC BY-NC-ND 4.0), which permits the noncommercial replication and distribution of the article with the strict proviso that no changes or edits are made and the original work is properly cited (including links to both the formal publication through the relevant DOI and the license). See: https://creativecommons.org/licenses/by-nc-nd/4.0/.

\section{References}

1. Rawla P, Sunkara T, Muralidharan P, et al. Update in global trends and aetiology of hepatocellular carcinoma. Contemp Oncol (Pozn) 2018;22:141-50.

2. Bray F, Ferlay J, Soerjomataram I, et al. Global cancer statistics 2018: GLOBOCAN estimates of incidence and mortality worldwide for 36 cancers in 185 countries. CA Cancer J Clin 2018;68:394-424.

3. Singal AG, El-Serag HB. Hepatocellular Carcinoma From
Epidemiology to Prevention: Translating Knowledge into Practice. Clin Gastroenterol Hepatol 2015;13:2140-51.

4. Baecker A, Liu X, La Vecchia C, et al. Worldwide incidence of hepatocellular carcinoma cases attributable to major risk factors. Eur J Cancer Prev 2018;27:205-12.

5. Bertot LC, Adams LA. Trends in hepatocellular carcinoma due to non-alcoholic fatty liver disease. Expert Rev Gastroenterol Hepatol 2019;13:179-87.

6. Llovet JM, Zucman-Rossi J, Pikarsky E, et al. Hepatocellular carcinoma. Nat Rev Dis Primers 2016;2:16018.

7. Ringelhan M, Pfister D, O'Connor T, et al. The immunology of hepatocellular carcinoma. Nat Immunol 2018;19:222-32.

8. Keenan BP, Fong L, Kelley RK. Immunotherapy in hepatocellular carcinoma: the complex interface between inflammation, fibrosis, and the immune response. J Immunother Cancer 2019;7:267.

9. Gao B, Jeong WI, Tian Z. Liver: An organ with predominant innate immunity. Hepatology 2008;47:729-36.

10. Krenkel O, Tacke F. Liver macrophages in tissue homeostasis and disease. Nat Rev Immunol 2017;17:306-21.

11. Wherry EJ. T cell exhaustion. Nat Immunol 2011;12:492-9.

12. Zhou G, Sprengers D, Boor PPC, et al. Antibodies Against Immune Checkpoint Molecules Restore Functions of Tumor-Infiltrating T Cells in Hepatocellular Carcinomas. Gastroenterology 2017;153:1107-19.e10.

13. Wu K, Kryczek I, Chen L, et al. Kupffer cell suppression of CD8+ T cells in human hepatocellular carcinoma is mediated by B7-H1/programmed death-1 interactions. Cancer Res 2009;69:8067-75.

14. Unitt E, Rushbrook SM, Marshall A, et al. Compromised lymphocytes infiltrate hepatocellular carcinoma: the role of T-regulatory cells. Hepatology 2005;41:722-30.

15. Jung HI, Jeong D, Ji S, et al. Overexpression of PD-L1 and PD-L2 Is Associated with Poor Prognosis in Patients with Hepatocellular Carcinoma. Cancer Res Treat 2017;49:246-54.

16. El-Khoueiry AB, Sangro B, Yau T, et al. Nivolumab in patients with advanced hepatocellular carcinoma (CheckMate 040): an open-label, non-comparative, phase $1 / 2$ dose escalation and expansion trial. Lancet 2017;389:2492-502.

17. Zhu AX, Finn RS, Edeline J, et al. Pembrolizumab in patients with advanced hepatocellular carcinoma 
previously treated with sorafenib (KEYNOTE-224): a non-randomised, open-label phase 2 trial. Lancet Oncol 2018;19:940-52.

18. Qin S, Ren Z, Meng Z et al. Camrelizumab in patients with previously treated advanced hepatocellular carcinoma: a multicentre, open-label, parallel-group, randomised, phase 2 trial. Lancet Oncol 2020;21:571-80.

19. Yau T, Park JW, Finn RS, et al. CheckMate 459: A randomized, multi-center phase III study of nivolumab (NIVO) vs sorafenib (SOR) as first-line (1L) treatment in patients (pts) with advanced hepatocellular carcinoma (aHCC). Ann Oncol 2019;30:874-5.

20. Finn RS, Ryoo BY, Merle P, et al. Pembrolizumab As Second-Line Therapy in Patients With Advanced Hepatocellular Carcinoma in KEYNOTE-240: A Randomized, Double-Blind, Phase III Trial. J Clin Oncol 2020;38:193-202.

21. Sangro B, Gomez-Martin C, de la Mata M, et al. A clinical trial of CTLA-4 blockade with tremelimumab in patients with hepatocellular carcinoma and chronic hepatitis C. J Hepatol 2013;59:81-8.

22. Duffy AG, Ulahannan SV, Makorova-Rusher O, et al. Tremelimumab in combination with ablation in patients with advanced hepatocellular carcinoma. J Hepatol 2017;66:545-51.

23. Chen DS, Mellman I. Oncology meets immunology: the cancer-immunity cycle. Immunity 2013;39:1-10.

24. Kudo M. Combination cancer immunotherapy with molecular targeted agents/anti-CTLA-4 antibody for hepatocellular carcinoma. Liver Cancer 2019;8:1-11.

25. Kelley RK, Abou-Alfa GK, Bendell JC, et al. Phase I/II study of durvalumab and tremelimumab in patients with unresectable hepatocellular carcinoma (HCC): Phase I safety and efficacy analyses. Proc Am Soc Clin Oncol 2017;35:4073.

26. Kelley RK, Sangro B, Harris WP, et al. Efficacy, tolerability, and biologic activity of a novel regimen of tremelimumab (T) in combination with durvalumab (D) for patients (pts) with advanced hepatocellular carcinoma (aHCC). J Clin Oncol 2020;38:4508.

27. Yau T, Kang YK, Kim TY, et al. Efficacy and Safety of Nivolumab Plus Ipilimumab in Patients With Advanced Hepatocellular Carcinoma Previously Treated With Sorafenib: The CheckMate 040 Randomized Clinical Trial. JAMA Oncol 2020;6:e204564.

28. Kudo M. Scientific Rationale for Combined Immunotherapy with PD-1/PD-L1 Antibodies and VEGF Inhibitors in Advanced Hepatocellular Carcinoma.
Cancers (Basel) 2020;12:1089.

29. Hegde PS, Wallin JJ, Mancao C. Predictive markers of anti-VEGF and emerging role of angiogenesis inhibitors as immunotherapeutics. Semin Cancer Biol 2018;52:117-24.

30. Pishvaian M J, Lee MS, Ryoo B, et al. Updated safety and clinical activity results from a phase Ib study of atezolizumab+ bevacizumab in hepatocellular carcinoma (HCC). Ann Oncol 2018;29:mdy424-028.

31. He AR, Numata K, Lee KH, et al. 986P Efficacy of atezolizumab (atezo)+ bevacizumab (bev) after disease progression with atezo monotherapy in patients with previously untreated, unresectable hepatocellular carcinoma (HCC). Ann Oncol 2020;31:S690.

32. Finn RS, Qin S, Ikeda M, et al. Atezolizumab plus Bevacizumab in Unresectable Hepatocellular Carcinoma. N Engl J Med 2020;382:1894-905.

33. Sonbol MB, Riaz IB, Naqvi SAA, et al. Systemic Therapy and Sequencing Options in Advanced Hepatocellular Carcinoma. A Systematic Review and Network Metaanalysis. JAMA Oncol 2020;6:e204930.

34. Zhu AX, Finn RS, Ikeda M, et al. A phase Ib study of lenvatinib (LEN) plus pembrolizumab (PEMBRO) in unresectable hepatocellular carcinoma (uHCC). J Clin Oncol 2020;38:4519.

35. Llovet JM, El-Khoueiry AB, Vogel A, et al. 1016TiP LEAP-012 trial in progress: Pembrolizumab plus lenvatinib and transarterial chemoembolization (TACE) in patients with intermediate-stage hepatocellular carcinoma (HCC) not amenable to curative treatment. Ann Oncol 2020;31:S702-3.

36. Vogel A, Müller DW, De Toni E, et al. 988P Safety analysis of the run-in phase (1st and 2 nd cohort) of the IMMUNIB trial (AIO-HEP-0218/ass)-An open-label, single-arm phase II study evaluating safety and efficacy of immunotherapy with nivolumab in combination with lenvatinib in advanced stage HCC. Ann Oncol 2020;31:S691.

37. Shen L, Zhang Y, Guo Y, et al. 987P A phase Ib study of the PD-1 antagonist CS1003 plus lenvatinib (LEN) in Chinese patients (pts) with the first-line (1L) unresectable hepatocellular carcinoma (uHCC). Ann Oncol 2020;31:S690-1.

38. Yau T, Zagonel V, Santoro A, et al. Nivolumab (NIVO) + ipilimumab (IPI) + cabozantinib (CABO) combination therapy in patients (pts) with advanced hepatocellular carcinoma (aHCC): Results from CheckMate 040. J Clin Oncol 2020;38:478.

39. Galle PR, Kim RD, Sung MW, et al. 990P Updated 
results of a phase Ib study of regorafenib (REG) plus pembrolizumab (PEMBRO) for first-line treatment of advanced hepatocellular carcinoma (HCC). Ann Oncol 2020;31:S691-2.

40. Jia F, Ren Z, Xu J, et al. 991P Sintilimab plus IBI305 as first-line treatment for advanced hepatocellular carcinoma. Ann Oncol 2020;31:S692.

41. Xu J, Zhang Y, Jia R, et al. Anti-PD-1 Antibody SHR-1210 Combined with Apatinib for Advanced Hepatocellular Carcinoma, Gastric, or Esophagogastric Junction Cancer: An Open-label, Dose Escalation and Expansion Study. Clin Cancer Res 2019;25:515-23.

42. Xu J, Shen J, Gu S, et al. 983P Camrelizumab (C) in combination with apatinib (A) in patients with advanced hepatocellular carcinoma (RESCUE): An open-label, multi-center, phase II trial. Ann Oncol 2020;31:S689.

43. June CH, O'Connor RS, Kawalekar OU, et al. CAR $\mathrm{T}$ cell immunotherapy for human cancer. Science 2018;359:1361-5.

44. Srivastava S, Riddell SR. Engineering CAR-T cells: Design concepts. Trends Immunol 2015;36:494-502.

45. Beckermann KE, Dudzinski SO, Rathmell JC. Dysfunctional $\mathrm{T}$ cell metabolism in the tumor microenvironment. Cytokine Growth Factor Rev 2017;35:7-14.

46. Scarfò I, Maus MV. Current approaches to increase CAR T cell potency in solid tumors: targeting the tumor microenvironment. J Immunother Cancer 2017;5:28.

47. Ninomiya S, Narala N, Huye L, et al. Tumor indoleamine 2,3-dioxygenase (IDO) inhibits CD19-CAR T cells and is downregulated by lymphodepleting drugs. Blood 2015;125:3905-16.

48. Davila ML, Riviere I, Wang X, et al. Efficacy and toxicity management of 19-28z CAR T cell therapy in B cell acute lymphoblastic leukemia. Sci Transl Med 2014;6:224ra25.

49. Bonifant CL, Jackson HJ, Brentjens RJ, et al. Toxicity and management in CAR T-cell therapy. Mol Ther Oncolytics 2016;3:16011.

Cite this article as: Uson Junior PLS, Liu AJ, Sonbol MB, Borad MJ, Bekaii-Saab TS. Immunotherapy and chimeric antigen receptor T-cell therapy in hepatocellular carcinoma. Chin Clin Oncol 2021;10(1):11. doi: 10.21037/cco-20-231
50. Gao H, Li K, Tu H, et al. Development of T cells redirected to glypican-3 for the treatment of hepatocellular carcinoma. Clin Cancer Res 2014;20:6418-28.

51. Jiang Z, Jiang X, Chen S, et al. Anti-GPC3-CAR T Cells Suppress the Growth of Tumor Cells in Patient-Derived Xenografts of Hepatocellular Carcinoma. Front Immunol 2017;7:690.

52. Li W, Guo L, Rathi P, et al. Redirecting T Cells to Glypican-3 with 4-1BB Zeta Chimeric Antigen Receptors Results in Th1 Polarization and Potent Antitumor Activity. Hum Gene Ther 2017;28:437-48.

53. Guo X, Jiang H, Shi B, et al. Disruption of PD-1 Enhanced the Anti-tumor Activity of Chimeric Antigen Receptor T Cells Against Hepatocellular Carcinoma. Front Pharmacol 2018;9:1118.

54. Mizukoshi E, Kaneko S. Immune cell therapy for hepatocellular carcinoma. J Hematol Oncol 2019;12:52.

55. McGowan E, Lin Q, Ma G, et al. PD-1 disrupted CAR-T cells in the treatment of solid tumors: Promises and challenges. Biomed Pharmacother 2020;121:109625.

56. Galle PR, Foerster F, Kudo M, et al. Biology and significance of alpha-fetoprotein in hepatocellular carcinoma. Liver Int 2019;39:2214-29.

57. Luo X, Cui H, Cai L, et al. Selection of a Clinical Lead TCR Targeting Alpha-Fetoprotein-Positive Liver Cancer Based on a Balance of Risk and Benefit. Front Immunol 2020;11:623.

58. Goyal L, Frigault M, Meyer T, et al. Initial safety of AFP SPEAR T-cells in patients with advanced hepatocellular carcinoma. Available online: https://cancerres.aacrjournals. org/content/79/13_Supplement/3183

59. Zhu W, Peng Y, Wang L, et al. Identification of $\alpha$ fetoprotein-specific T-cell receptors for hepatocellular carcinoma immunotherapy. Hepatology 2018;68:574-89.

60. Docta RY, Ferronha T, Sanderson JP, et al. Tuning T-Cell Receptor Affinity to Optimize Clinical Risk-Benefit When Targeting Alpha-Fetoprotein-Positive Liver Cancer. Hepatology 2019;69:2061-75. 